\title{
$Q$-Factor and Frequency Shift of Resonating Silicon Diaphragms in Air
}

ALBERT PRAK, FRANS R. BLOM*, MIKO ELWENSPOEK and THEO S. J. LAMMERINK

Department of Electrical Engineering, University of Twente, P.O. Box 217, 7500 AE Enschede (The Netherlands)

\begin{abstract}
The dependence of the relative frequency shift and the $Q$-factor of the first two modes of vibration of square silicon diaphragms on the diaphragm geometry and the air pressure is investigated. The experimental results are compared with the theory, which is based on Lamb's theory for circular diaphragms. It assumes acoustic radiation to be the determining mechanism for the energy loss of the diaphragm. The experimental results for both the frequency shift and the $Q$-factor as a function of the pressure deviate from the theory. A possible explanation for this deviation is the assumption that viscous damping plays an important role. It is found that the vibrating diaphragm is able to put the whole wafer into vibration, which causes several interfering effects. This phenomenon limits the applicability of vibrating diaphragms in resonant sensors.
\end{abstract}

\section{Introduction}

In this paper the properties of the transverse vibrations of silicon diaphragms, which are applied in silicon resonant microsensors, are studied. In such sensors, the resonance frequency of the diaphragm is determined by the quantity to be measured, e.g., a pressure difference $[1,2]$ or a mass flow [3]. The properties investigated consist of three quantities, namely the resonance frequency in vacuum $\left(f_{\mathrm{v}}\right)$, the relative shift of

\footnotetext{
*Present address: Océ Nederland B.V., Venlo, The Netherlands.
}

this resonance frequency $\left.\left(r f_{s}=\left(f_{\mathrm{r}}-f_{\mathrm{v}}\right) / f_{\mathrm{v}}\right)\right)$, which is observed when the diaphragm is submerged in a fluid, and the quality factor $(Q)$, which is related to the energy loss of the vibrating diaphragm. The dependence of these three quantities on the diaphragm geometry and the air pressure is studied both theoretically and experimentally. This is done for the first two modes of vibration. The aim of the research is to obtain design rules for optimizing diaphragm geometry for applications in resonant sensors. A similar study has been carried out in our group for vibrating cantilever beams [4].

\section{Theory}

\section{The First Mode of Vibration}

The resonance frequency of a clamped, square plate without residual stress is well known, e.g., [5]:

$f_{0,1}=1.654 c_{\mathrm{p}} h / a^{2}$

with $c_{\mathrm{p}}$ the speed of sound in the plate material $\left(c_{\mathrm{p}}^{2}=E / \rho_{\mathrm{p}}\left(1-\mu^{2}\right)\right), h$ the thickness of the plate, $a$ the length of an edge, $E$ Young's modulus, $\rho_{\mathrm{p}}$ the density and $\mu$ Poisson's ratio. Subscripts 0 and 1 refer to zero residual stress and the first mode respectively.

From the calculation of the impedance of a vibrating plate element (i.e., the ratio of the force exerted by the element on the fluid to the velocity of the element) a complex expression results whose imaginary part is related to an increase of the inertia of the plate element [6]. The total effect is equivalent to increasing the mass of the plate by a relative amount $\beta=T_{\mathrm{f}} / T_{\mathrm{p}}$, with $T_{\mathrm{f}}$ and $T_{\mathrm{p}}$ the kinetic energies

(C) Elsevier Sequoia/Printed in The Netherlands 
of the fluid and the plate respectively. For a square plate the result is [7]

$\beta_{1}=0.6904 \frac{\rho_{\mathrm{f}} a}{\rho_{\mathrm{p}} h}$

with $\rho_{\mathrm{f}}$ the density of the fluid. The viscosity of the fluid is neglected in this calculation. We find

$r f s_{1}=\left(\frac{1}{1+\beta_{1}}\right)^{1 / 2}-1 \approx-\frac{\beta_{1}}{2}\left(\beta_{1} \ll 1\right)$

The relative frequency shift due to damping $\left(r f s_{1}=-1 / 4 Q_{1}^{2}\right)$ is neglected.

The quality factor $Q$ can be calculated with the following expression:

$Q=2 \pi \frac{\text { total energy of the system }}{\text { dissipated energy per cycle }}$

The total energy of the system equals the maximum kinetic energy of the plate plus the maximum kinetic energy of the surrounding fluid. Since the vibration is nearly perpendicular to all surface elements, the only pressuredependent damping mechanism to be considered is energy loss by acoustic radiation. Viscous damping is neglected. In a manner completely analogous to Lamb's calculations for circular plates [8], but with a mode shape $w(x, y)=\left(1-4 x^{2} / a^{2}\right)\left(1-4 y^{2} / a^{2}\right)$, we find

$Q_{1}=6.415 \frac{\rho_{\mathrm{p}} c_{\mathrm{f}} h}{\rho_{\mathrm{f}} 2 \pi f a^{2}}\left(1+\beta_{1}\right)$

with $c_{\mathrm{f}}$ the speed of sound in the fluid. If the plate obeys eqn. (1), we can substitute eqn. (1) into eqn. (4) to obtain a value for $Q_{1}$ in resonance. If for some reason (e.g., stress) the plate does not obey eqn. (1) and has a resonance frequency in vacuum $f_{\mathrm{v}, 1}=R_{1} f_{0,1}$, we can write

$Q_{1}=0.617 \frac{\rho_{\mathrm{p}} c_{\mathrm{f}}}{\rho_{\mathrm{r}} c_{\mathrm{p}}} \frac{\left(1+\beta_{1}\right)^{3 / 2}}{R_{1}}$

Besides this acoustic damping, there exists a pressure-independent damping component as a result of internal friction in the crystal lattice and energy radiation into the bulk material at the edges of the plate. This structural damping component provides an upper value for $Q_{1}$, say $Q_{\mathrm{str}, 1}$, which can never be exceeded.

\section{The Second Mode of Vibration}

In this mode of vibration there is a nodal line parallel to the edge, which divides the plate into two equal parts [5]. These parts vibrate with opposite phases. For symmetry reasons, it follows that there are two secondmode vibrations. Theoretically these two resonances have exactly the same frequency; however, in practice they will differ slightly due to the inhomogeneity of the diaphragm. The resonance frequency is [5]

$f_{0.2}=2.040 f_{0.1}=3.374 h c_{\mathrm{p}} / a^{2}$

If the above results for $Q_{1}$ and $r s_{s_{1}}$ are compared with the results for circular plates [8], the conclusion is that the shape of the plate is only of minor influence. The deviations are much smaller than the failures as a result of the accuracy in the thickness determination of the diaphragms, for example. The comparison is carried out by replacing the diameter of the circular plates by the length of the edge of the square plates. If we assume this similarity between circular and square plates to be true for the second mode of vibration as well, we find, starting with Lamb's results for the second mode of a circular plate [8], the following expressions for $\beta_{2}$ :

$\beta_{2}=0.3087 \frac{\rho_{\mathrm{f}} a}{\rho_{\mathrm{p}} h}$

A relation like eqn. (3) holds for $r f_{s_{2}}$. For $Q_{2}$ we find

$$
\begin{aligned}
Q_{2} & =\frac{55296}{120} \frac{\rho_{\mathrm{p}} h}{\rho_{\mathrm{f}} a^{4}} \frac{c_{f}^{3}}{(2 \pi f)^{3}}\left(1+\beta_{2}\right) \\
& =0.0284 \frac{\rho_{\mathrm{p}} a^{2} c_{\mathrm{f}}^{3}}{\rho_{\mathrm{f}} h^{2} c_{\mathrm{p}}^{3}} \frac{\left(1+\beta_{2}\right)^{5 / 2}}{R_{2}^{3}}
\end{aligned}
$$

$R_{2}$ is defined like $R_{1}$.

\section{Application: a Silicon Diaphragm in Air}

The previous results are now applied to a silicon diaphragm vibrating in air of $293 \mathrm{~K}$. The following constants are used: $E=$ $1.55 \times 10^{11} \mathrm{~Pa}, \quad \mu=0.06, \quad \rho_{\mathrm{p}}=2330 \mathrm{kgm}^{-3}$, $c_{\mathrm{p}}=8160 \mathrm{~m} \mathrm{~s}^{-1}, \rho_{\mathrm{f}}=1.18 \times 10^{-5} \mathrm{p} / p_{\mathrm{0}} \mathrm{kg} \mathrm{m}^{-3}$ $\left(p_{0}=1 \mathrm{~Pa}\right), c_{\mathrm{f}}=343 \mathrm{~m} \mathrm{~s}^{-1} . c_{\mathrm{f}}$ is independent 
TABLE 1. Theoretical expressions for the relative additive mass $\beta$, the relative frequency shift $r f s$ and the quality factor $Q$ for the first two modes of vibration. $p_{0}=1 \mathrm{~Pa}$

\begin{tabular}{lll}
\hline General & $\beta \ll 1$ & $\beta \gg 1$ \\
\hline$\beta_{1}=3.50 \times 10^{-9} \frac{a p}{h p_{0}}$ & $r s_{1}=-1.75 \times 10^{-9} \frac{a p}{h p_{0}}$ & $r f s_{1}=-1$ \\
$Q_{1}=\frac{5.12 \times 10^{6}}{R_{1}} \frac{p_{0}}{p}\left(1+\beta_{1}\right)^{3 / 2}$ & $Q_{1}=\frac{5.12 \times 10^{6}}{R_{1}} \frac{p_{0}}{p}$ & $Q_{1}=\frac{1.05 \times 10^{-6}}{R_{1}}\left(\frac{a}{h}\right)^{3 / 2}\left(\frac{p}{p_{0}}\right)^{1 / 2}$ \\
$\beta_{2}=1.56 \times 10^{-9} \frac{a p}{h p_{0}}$ & $r s_{2}=-7.82 \times 10^{-10} \frac{a p}{h p_{0}}$ & $r f s_{2}=-1$ \\
$Q_{2}=\frac{4.16 \times 10^{2}}{R_{2}^{3}}\left(\frac{a}{h}\right)^{2} \frac{p_{0}}{p}\left(1+\beta_{2}\right)^{5 / 2}$ & $Q_{2}=\frac{4.16 \times 10^{2}}{R_{2}^{3}}\left(\frac{a}{h}\right)^{2} \frac{p_{0}}{p}$ & $Q_{2}=\frac{4.02 \times 10^{-20}}{R_{2}^{3}}\left(\frac{a}{h}\right)^{9 / 2}\left(\frac{p}{p_{0}}\right)^{3 / 2}$
\end{tabular}

of the pressure. With the expressions (2), (3), (5), (7) and (8), the results presented in Table 1 are obtained. The Table consists of three columns. In the first column the general expressions are given, in the second the expressions when $\beta \ll 1$ (this condition was always fulfilled in our experiments), and in the last one the expressions when $\beta \gg 1$ (in practice these expressions are not very useful because $f_{r}$ becomes very small). For a silicon diaphragm in air of 1 bar we find $\beta_{1}=1$ for $a / h=2860$. The results for a diaphragm with $a / h=1000$ are presented in Fig. 1 .

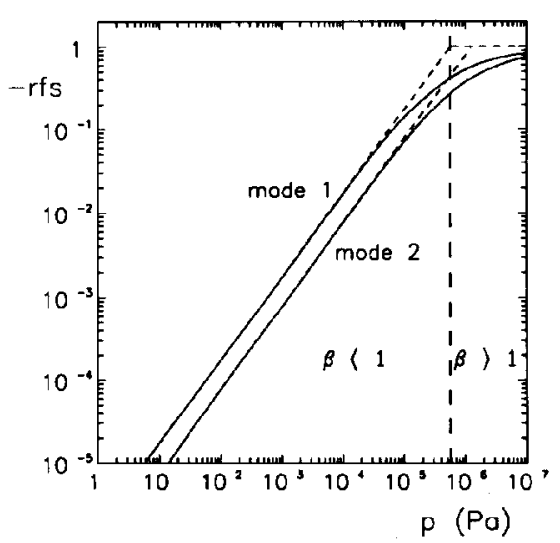

(a)

\section{Experimental}

Since the purpose of the study was the understanding of the behaviour of pure silicon diaphragms, both excitation and detection of the vibrations were done externally, i.e., no active films were deposited on the diaphragm. Excitation was done optothermally with a $830 \mathrm{~nm}, 30 \mathrm{~mW}$ laser diode. Optothermal excitation causes an inhomogeneous d.c. heating of the diaphragm, which could influence its behaviour. However, experiments with varying laser power showed

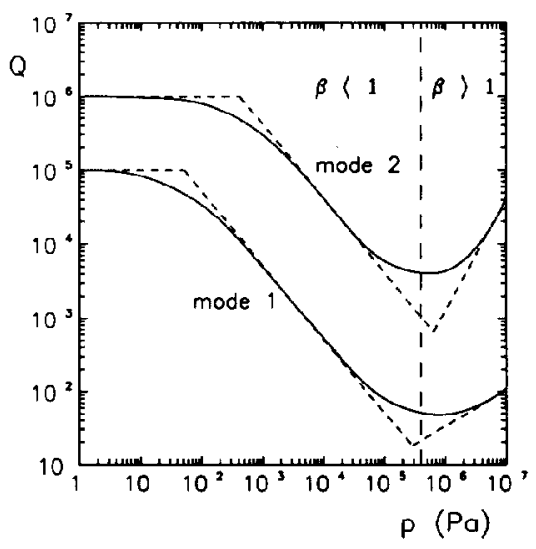

(b)

Fig. 1. Theoretical results for $r f s(p)$ (a) and $Q(p)$ (b) for the first two modes of a square silicon diaphragm vibrating in air of $293 \mathrm{~K}$. The following parameters are chosen (arbitrary values): $a / h=1000, Q_{\text {str, } 1}=10^{5}$ and $Q_{\text {str, } 2}=10^{6}$. In both graphs, the dashed lines represent the asymptotes for $\beta \ll 1$ and $\beta \gg 1$ respectively (see Table 1). The dashed vertical lines correspond to $\beta=1$. At very low pressures, the $Q$ values are determined by their structural values, which are presented by the horizontally dashed lines in (b). 
that this influence is very small (if measurable at all). Detection of the vibration was done by means of a Michelson interferometer with which vibration amplitudes down to a few pm can be measured. The sample was placed in a bell jar in which the pressure could be varied between 1 and $10^{5} \mathrm{~Pa}$.

The diaphragms were fabricated by wet chemical etching in aqueous $\mathrm{KOH}$. Diaphragms were made with sides of 4,8 and $16 \mathrm{~mm}$ and various thicknesses ranging from 3 to $40 \mu \mathrm{m}$. The diaphragms thinner than $10 \mu \mathrm{m}$ were etched electrochemically. Etching was stopped on a low n-doped epitaxial layer. In order to obtain a well-defined electric contact, which is necessary in the electrochemical etch stop process, a thin (approximately $0.3 \mu \mathrm{m}$ ) highly doped p-layer was diffused into the low n-doped epitaxial layer. For the diaphragms thicker than $10 \mu \mathrm{m}$ a simple time stop was used. In this way diaphragms with very low stress were obtained. We used 2 inch, $280 \mu \mathrm{m},\langle 100\rangle$ wafers. The surface roughness of all diaphragms was less than $1 \mu \mathrm{m}$; however, a change of thickness of several $\mu \mathrm{m}$ over the entire area has been observed at the time-stopped diaphragms. Two sample series were fabricated: one with multiple diaphragms per wafer and the other with a single diaphragm per wafer.

We used different methods in order to mount the wafer to its surroundings. The first one is to fold a piece of silicone rubber around the flat of the wafer which is clamped into a metal holder. We call this the soft wafer-to-support mounting. The second one is to clamp the wafer into the metal holder directly. This is called the rigid wafer-to-support mounting.

\section{Results and Discussion}

\section{The Spectra}

Before we present the results of $f_{\mathrm{v}}, r f_{s}$ and $Q$, we first pay attention to the frequency spectra of the diaphragms. A typical spectrum is plotted in Fig. 2. This spectrum is of a diaphragm from the first sample series (i.e., with more diaphragms per wafer). The dimensions of the diaphragm are $a=8 \mathrm{~mm}$ and $h=12 \mu \mathrm{m}$. The resonance marked by 1 is the first-mode resonance (expected value $2530 \mathrm{~Hz}$, observed $2520 \mathrm{~Hz}$ ). At $5600 \mathrm{~Hz}$ we see the two second-mode resonances (marked by 2 , expected value $5160 \mathrm{~Hz}$ ).

$\mathrm{Up}$ to now the spectrum has met our expectations. However, we observe a number of other resonances. Peaks 3 and 4 (at 1300 and $1680 \mathrm{~Hz}$ ) are due to mechanical crosstalk between the diaphragm under consideration and a nearby $16 \times 16 \mathrm{~mm}$ diaphragm. It was found from interferometric observations that the whole wafer is put into vibration by the vibrations of the excited diaphragm. This wafer vibration serves as an excitation for other diaphragms, e.g., the $16 \times 16 \mathrm{~mm}$ diaphragm. The $16 \times 16 \mathrm{~mm}$ diaphragm, in turn, influences the wafer vibration and thus vibrations of the $8 \times 8 \mathrm{~mm}$ diaphragm, which is observed in the spectrum of the latter. We first see an amplification of the vibration at one of the resonance frequencies (first or higher mode) of the $16 \times 16 \mathrm{~mm}$

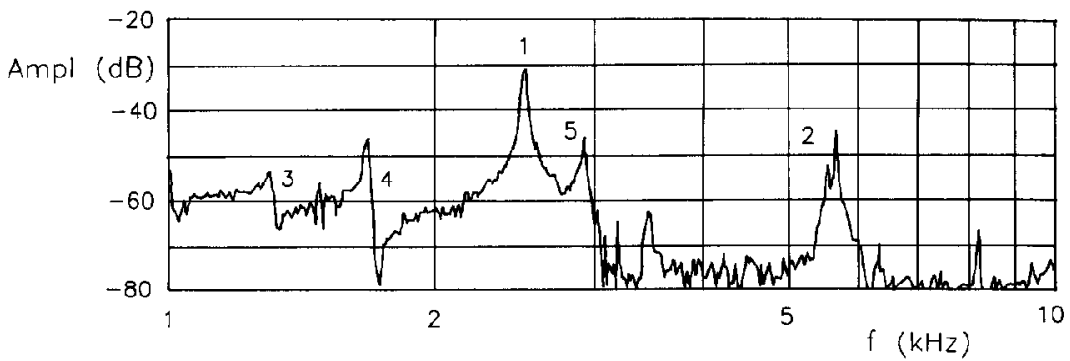

Fig. 2. Spectrum of a diaphragm with $a=8 \mathrm{~mm}$ and $h=16 \mathrm{~mm}$. Origin of the resonances: 1 , first-mode resonance; 2, second-mode resonances; 3 and 4 , mechanical cross-talk with other diaphragms; and 5, origin unknown. 


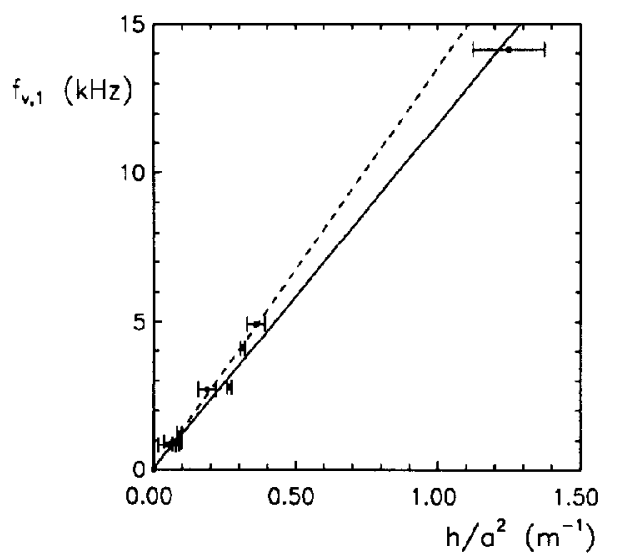

Fig. 3. $f_{\mathrm{v}, 1}$ as a function of the parameter $h / a^{2}$. Solid line: experiment, dashed line: theory.

diaphragm, immediately followed by an attenuation. This pattern is typical for a set of coupled oscillators.

Frequently a peak on the right flank of the first-mode resonance was observed. In Fig. 2 this peak is marked by 5 . The origin of this peak is not yet understood.

\section{The First Mode of Vibration}

A graph of $f_{\mathrm{v}, 1}$ versus $h / a^{2}$ for various diaphragms is presented in Fig. 3. The error bars result from the uncertainty in the diaphragm thickness. A linear least-square fit through the origin gives $f_{\mathrm{v}, 1} a^{2} / h=11.6 \times 10^{3}$. With $c_{\mathrm{f}}=8160 \mathrm{~m} \mathrm{~s}^{-1}$ we find with eqn. (1) that $f_{\mathrm{v}, 1}=1.42 / 1.65 f_{0,1}=0.86 f_{0,1}$. This lowering of $f_{0,1}$ may result from the fact that in practice we do not have clamped edges (as the model assumes), but boundary conditions somewhere between clamped and simply supported (in the case of a simply supported plate the proportionality constant in eqn. (1) is 0.91). In measurements concerning the diaphragms with $h<10 \mu \mathrm{m}$ we found large deviations between $f_{\mathrm{v}, 1}$ and $f_{0,1}$. These deviations probably result from the stress which is introduced in the fabrication process. These measurements are not plotted in Fig. 3. Further we found that the stress as a result of mounting the wafer to its surroundings has considerable influence on $f_{\mathrm{v}, 1}$. Fluctuations of $10 \%$

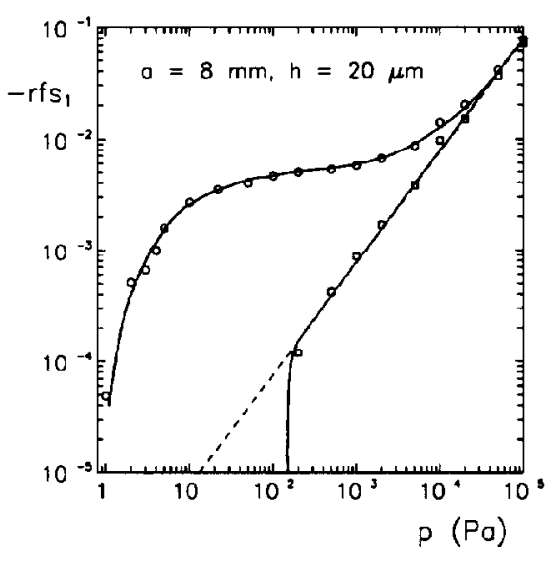

Fig. 4. Typical experimental $r s_{1}(p)$ curves (solid lines). $O: f_{v, 1}=4102.0 \mathrm{~Hz}$ and $\square: f_{v, 1}=4082.1 \mathrm{~Hz}$. The dashed line represents the theoretical curve.

or more have been observed for the rigid wafer-to-support mounting.

In Fig. 4 the experimental and theoretical curves for $r f s_{1}$ as a function of $p$ are plotted of a diaphragm with $a=8 \mathrm{~mm}$ and $h=$ $20 \mu \mathrm{m}$. The curve with measurements marked $O$ shows results for $f_{\mathrm{v}, 1}=4102.0 \mathrm{~Hz}$, the value which we actually measured. This curve shows large deviations from theory over a broad pressure range. This phenomenon was observed for all samples and is very reproducible.

However, if $f_{\mathrm{v}, \mathrm{I}}$ is put equal to $4082.1 \mathrm{~Hz}$, the measurements marked $\square$ are found. This curve agrees very well with the theory. At pressures below approximately $150 \mathrm{~Pa}$, the assumed $f_{\mathrm{v}, 1}$ leads to positive values of $r s_{1}$, i.e., a negative additive mass. The pressure at which this phenomenon occurs is the same for all samples. As a qualitative explanation for this artificial matching of experiment and theory, the desorption of material from the silicon surface at pressures below $150 \mathrm{~Pa}$ could be adduced. The new $f_{\mathrm{v}, 1}$ of $4082.1 \mathrm{~Hz}$ must be interpreted as the resonance frequency in vacuum if no desorption had occurred. Quantitatively, however, the effect is too large. Desorption of a homogeneously spread mass of $27 \mu \mathrm{g}$ is needed to account for the change of the vacuum resonance frequency. This corresponds to a few hundred 


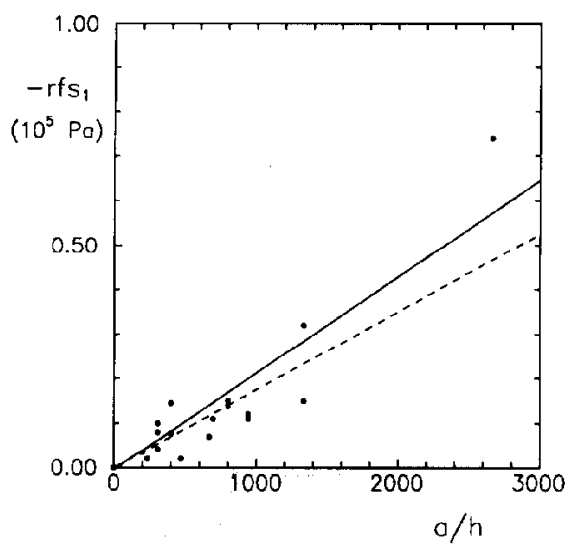

Fig. 5. $r f s_{1}$ as a function of the parameter $a / h$ at $p=10^{5} \mathrm{~Pa}$ for various diaphragms. Solid line: experiment, dashed line: theory.

monolayers of $\mathrm{N}_{2}$ or $\mathrm{H}_{2} \mathrm{O}$, a value which is not very plausible.

Another possible reason for the measured $r f s_{1}(p)$ curve is the following. In [4] it is shown that, for beams, the function $r f s(p)$ depends on the dominating damping mechanism, for example $r f s \approx p$ in one pressure region and $r f s \approx \sqrt{ } p$ in another. As will be reported below, other damping mechanisms probably play a role besides the acoustic radiation. This means the the dependence of $r f s$ on $p$ can deviate from the theory presented above.

In Fig. 5 the value of $r f s_{1}$ at $p=10^{5} \mathrm{~Pa}$ is plotted versus the ratio $a / h$ for various diaphragms. The large dispersion results from the uncertainty in the thickness of the diaphragms. The theoretical value for the slope of the graph equals $-1.75 \times 10^{-4}$ (Table $1, \beta \ll 1)$. A least-square linear fit through the origin gives a value of $-2.2 \times 10^{-4}$, which agrees with the theory within the experimental uncertainty.

A typical $Q_{1}(p)$ measurement is shown in Fig. 6. The results are plotted for two different wafer-to-support mountings of a diaphragm with $a=16 \mathrm{~mm}$ and $h=16 \mu \mathrm{m}$. The way in which the wafer is mounted to the support appeared to be the determining factor for the structural $Q$-factor. Evidently, energy is transported to the support through

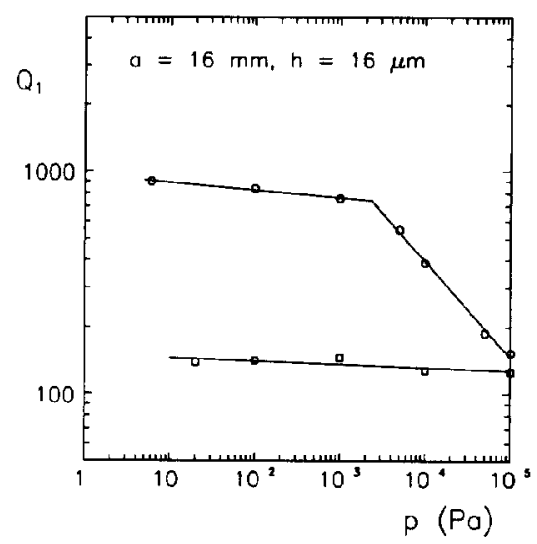

Fig. 6. Experimental results for $Q_{1}$ as a function of $p$ for two different wafer-to-support mountings of a diaphragm with $a=16 \mathrm{~mm}$ and $h=16 \mu \mathrm{m}$. O: rigid wafer-to-support mounting and $\square$ : soft wafer-to-support mounting.

the wafer. This is in agreement with the appearance of the mechanical cross-talk between diaphragms, which we reported above. The more rigidly the wafer is mounted, the less energy is transported ( $Q_{\text {str }}$ increases). This is a very important conclusion with respect to the packaging of sensors with vibrating diaphragms. From our measurements it was found that for equally clamped diaphragms the structural $Q$-factor tends to increase as $h$ decreases. This suggests that the structural $Q$-factor depends on the mass ratio of wafer and diaphragm. In general, a pressure-dependent $Q$-factor in the high-pressure region of rigidly mounted wafers is observed. We tried to fit the experimental results in this region as $\left(Q_{1}(p) \approx p^{-1}\right.$, but these fits were not satisfactory. In Fig. 6 the exponent $e$ in the relation $Q_{1}=p^{-e}$ has the value 0.4 . We found experimental values for $e$ between 0.1 and 0.8 . The exponent $e$ tends to increase as the dimensions of the diaphragm decrease. This suggests a direct relation between the mass of the diaphragm and the exponent $e$. The theoretical value based on the acoustic model $(e=1)$ was never observed. This could mean that another damping mechanism is dominant. One possibility is, as . Lamb suggests [8], that viscous processes at the rim of 
the etched cavity which forms the diaphragm play a role.

\section{The Second Mode of Vibration}

The ratio of the resonance frequencies of the second and the first mode was experimentally determined to be between 1.5 and 2.4. The scattering around the theoretical value 2.040 (eqn. (6)) may be caused by stress introduced by the wafer-to-support mounting.

The experimental results for $r s_{2}$ as a function of $p$ show the same behaviour as for the first mode. Consequently, the same reasons as for the first mode can be adduced to explain the deviations from the theory.

The experimentally determined $Q_{2}$ values are always smaller than the theoretical ones. The pressure dependence is, just as for the first mode, less strong than the acoustic model predicts. These two observations make us suspect that another damping mechanism is also dominant for the second mode over the acoustic mechanism.

\section{Conclusions}

Generally, we found poor agreement between Lamb's theory for the pressure dependence of the resonance frequency and the $Q$-factor of square vibrating diaphragms in air. Attempts to ascribe the deviation of the experimental $r f s(p)$ to the desorption of gas or water are qualitatively successful, but fail because several hundreds of monolayers are required to account for the effect. An alternative explanation for the observed deviation from the theory is the inclusion of viscous damping. In principle, viscous damping affects both the $Q$-factor and additive mass [9]. However, viscous damping is related to normal gradients in the velocity of the streaming fluid, which intuitively can be neglected in the problem described here. To our knowledge there is no model available which includes shear viscosity in the damping of transversely vibrating plates. Such models have to be developed in order to verify if viscosity really can account for the observed effects. Viscous processes at the rim of the diaphragm have to be included in the model as well.

Great attention must be paid to the strong influence of the mounting on the $Q$-factor and to the considerable cross-talk between diaphragms on the same wafer. These effects demonstrate that the coupling of the vibrating diaphragm to the wafer is very strong. The performance of a device based on the shift of the resonance frequency of a diaphragm, such as the devices described in refs. $1-3$, sensitively depends on how the diaphragm is mounted. Not only is the resonance frequency very sensitive to stress (e.g., packaging or thermal stress), but also the $Q$-factor depends on how the diaphragm is mounted to the rest of the device and on the total mass of the device; the $Q$-factor is directly related to the precision with which the resonance frequency can be determined. These results make the feasibility of large vibrating diaphragm sensors questionable.

\section{Acknowledgements}

The authors would like to thank Dick Ekkelkamp and Robert Bakker for their contribution to the experimental work.

\section{References}

I J. G. Smits, H. A. C. Tilmans, K. Hoen, H. Mulder, J. van Vuuren and G. Boom, Resonant diaphragm pressure measurement system with $\mathrm{ZnO}$ on Si excitation, Sensors and Actuators, 4 (1983) 565-571.

2 T. S. J. Lammerink and S. J. Gerritsen, Fiber-optic sensors based on resonating mechanical structures, Proc. Int. Conf. Fiber Optic Sensors II, The Hague, The Netherlands, SPIE Proc., 798 (1987) 67-70.

3 S. Bouwstra, P. Kemna and R. Legtenberg, Thermally excited resonating membrane mass flow sensor, Sensors and Actuators, 20 (1989) 213-233.

4 F. R. Blom, S. Bouwstra, M. Elwenspoek, J. H. J. Fluitman, Dependence of the quality factor of micromachined silicon beam resonators on pressure and geometry, J. Vac. Sci. Technol, submitted for publjcation.

5 D. Young, Vibrations of rectangular plates by the Ritz method, J. Appl. Mech., I7 (1950) 448-453. 
6 F. Montenero de Espinosa and J. A. GallegoJuárez, On the resonance frequencies of waterloaded circular plates, J. Sound Vib., 94 (1984) 217222.

7 R. D. Blevins, Formulas for Natural Frequency and Mode Shape, Krieger, Malaba, FL, 1987, p. 418.
$8 \mathrm{H}$. Lamb, On the vibrations of an elastic plate in contact with water, Proc. R. Soc. London Ser. A, 98 (1920) 205-216.

9 L. D. Landau and E. M. Lifschitz, Lehrbuch der theoretischen Physik, Vol. VI, Akademie Verlag, Berlin, 3rd edn, 1974, p. 97 ff. 